\title{
UMA INTERPRETAÇÃO DA LEI KALDOR-VERDOORN PARA A ANÁLISE SETORIAL DO PIB, PRODUTIVIDADE E EM- PREGO NA ECONOMIA BRASILEIRA ${ }^{1}$
}

\author{
Henrique Tomé da Costa Mata ${ }^{2}$ \\ Niraldo José Ponciano ${ }^{3}$ \\ Paulo Marcelo de Souza
}

\begin{abstract}
Resumo - Importantes indicadores de desempenho de qualquer economia são a dinâmica do mercado de trabalho e a distribuição de renda setorial, que estão relacionadas, direta e indiretamente, com a produtividade. Não obstante às restrições inerentes ao uso de diferentes conceitos de produtividade, Kaldor e Verdoorn investigaram relações entre produção, emprego e produtividade, visando explicar a dinâmica econômica inter-regional e intersetorial, cujas proposições resultaram na designação da Lei Kaldor-Verdoorn. O modelo que expressa tal Lei conjetura as relações entre crescimento da produtividade do trabalho e emprego em relação ao crescimento da renda, fornecendo medidas de impacto e de retornos à escala, particularmente aplicável ao setor industrial. Os fundamentos teóricos intrínsecos ao modelo foram desenvolvidos neste artigo, com aplicações empíricas às séries de PIB e emprego na economia brasileira, apoiando as hipóteses de Kaldor e Verdoorn quanto às estimativas das elasticidades obtidas.
\end{abstract}

Palavras-Chave: PIB, produtividade, emprego, lei Kaldor-Verdoorn.

\footnotetext{
Os autores agradecem aos pareceristas anônimos e, especialmente, ao editor da Revista de Economia e Agronegócio, pelas pertinências das considerações críticas efetuadas tanto na estrutura textual quanto no conteúdo do artigo. Recebido em 08/08/2005 Aceito em 10/11/2005.

2 Professor da Universidade Estadual de Santa Cruz - UESC. E-mail: hnrmata@uesc.br

3 Professor da Universidade Estadual do Norte Fluminense - UENF. E-mail: ponciano@uenf.br

4 Professor da Universidade Estadual do Norte Fluminense - UENF. E-mail: pmsouza@uenf.br
} 


\section{Introdução}

Nos anos iniciais da década de 90, a economia brasileira sofreu grande impacto tecnológico e de reestruturação produtiva que resultou no cenário de abertura comercial e exigências da competitividade externa. Os segmentos agropecuários, industriais e de serviços, ao absorverem as exigências do novo ambiente macroeconômico, tiveram efeitos que resultaram na variação temporal do PIB, emprego e produtividade. A descrição das relações características desses três agregados econômicos fundamentais da macroeconomia, essencialmente o comportamento da produtividade em relação ao emprego e o mercado de trabalho, a produção e outros aspectos ligados ao crescimento econômico, constitui a chave fundamental à formulação e análise de políticas econômicas. Segundo Vieira (1999), a busca de redução nos custos de produção e de aumento na qualidade, exigida pelo novo padrão de competitividade externa, desencadeou um processo acelerado de adoção de tecnologias poupadoras de trabalho, mediante adoção de novos métodos administrativos que resultou no acréscimo do desemprego, diminuindo a participação do setor industrial na geração de emprego. Gonçalves et al. (2003) assinalaram que a economia brasileira, no período de 1985-90, foi marcada pela recuperação dos níveis da produção e pela elevação da produtividade, após a fase do declínio econômico que caracterizou a primeira metade dos anos 80 . Entretanto, a partir dos anos 90, a abertura econômica e comercial que se seguiu ao Plano Collor foi marcada por trajetória ascendente da produtividade. A elevação na produtividade, neste período, foi conseqüência da abertura e do Programa Qualidade e Produtividade, que incentivou a adoção de técnicas novas, desestatização e desregulamentação da economia. A reestruturação do sistema produtivo visou à manutenção das atividades produtivas, ameaçadas pelo acesso de produtos estrangeiros competitivos, e à busca da produtividade, mediante redução dos custos e utilização de processos eficientes. A combinação de tendências de redução no emprego com manutenção da produção implicou a elevação da produtividade do trabalho nos anos 90 . 
A análise da produtividade ganha importância crescente no debate econômico, pois ela tem sido um dos principais instrumentos a possibilitar aumento da participação setorial na economia, com efeitos determinantes na distribuição de renda intra e intersetorial de um país. Normalmente, usam-se dois conceitos de produtividade - a do trabalho e a total dos fatores de produção, entre outros, conforme o tipo específico de fatores de produção em análise. A produtividade do trabalho descreve a relação entre cada nível de produção e o trabalho necessário a este nível, sendo mais indicada quando o interesse residir na análise do bem-estar econômico. A segunda é a relação entre produção e fatores humanos e físicos, ou seja, leva em conta os custos de capital e trabalho, entre outros fatores determinantes do arranjo tecnológico e social. Portanto, trata-se de um conceito adequado à análise da eficiência econômica geral de um sistema.

Neste trabalho, recorreu-se ao conceito da produtividade média de trabalho, em relação ao número total de empregos por setor e ao respectivo PIB setorial. Porém, a literatura levanta restrições a considerar dados agregados sobre produção e emprego e, portanto, sobre produtividade, pois o alto nível de agregação pode gerar dúvidas acerca da variação da produtividade e sua determinação exógena ou endógena ao nível setorial. Essas questões constituíram pontos-chaves nas reflexões de Verdoorn (1980), que estabeleceu os fundamentos teóricos para uma relação empírica, de longo prazo, entre crescimento da produtividade e crescimento da produção industrial; por exemplo, o que efetivamente ocorre, quando existem excesso de oferta de mão-de-obra e escassez de estoque de capital, como demonstrou a maioria das economias em desenvolvimento. Nesse aspecto, a Lei de Kaldor oferece uma boa estrutura para análise do comportamento da produtividade. $\mathrm{O}$ argumento de Kaldor $(1968,1975)$ é de que, se parte importante do crescimento da produtividade for endógena ao crescimento econômico, ilustra-se o significado da economia de escala no processo de desenvolvimento econômico, que é um pressuposto básico da estrutura teórica de Verdoorn, fundamentado no processo de crescimento cumulativo. A interpretação de Kaldor, para entender o comportamento da taxa de crescimento da 
economia nos países desenvolvidos, consiste de suas observações sobre as dimensões de aumento no nível do produto setorial e as necessidades de emprego e produtividade de trabalho, simultaneamente. Na versão simples de Verdoorn, estabelece-se uma relação positiva, de longo prazo, entre a taxa de crescimento da produtividade e a taxa de crescimento da renda, principalmente ao nível do setor industrial (Pieper, 2001).

Estudos de Salgado (2000) apontam várias causas para explicar flutuações indesejáveis da economia, quais sejam, o declínio na formação do estoque de capital, a alteração na estrutura da força de trabalho no setor de produção de bens capital para setores de serviços e outros segmentos de baixa produtividade, a ausência de progresso tecnológico e a extrema regulação econômica. A proposta definida neste artigo consiste dos seguintes objetivos: desenvolver a abordagem teórica na linha das proposições inerentes à Lei Kaldor-Verdoorn (KV) e sua interpretação aplicada à economia brasileira; estimar e analisar as elasticidades, de curto e longo prazo, da produtividade em relação ao crescimento de produto setorial da economia; analisar o comportamento do emprego, da produtividade, do PIB e do valor adicionado ao nível de três setores da economia.

A estrutura geral do artigo apresenta-se da seguinte forma. Uma seção introdutória, em que se discorre sobre alguns aspectos teóricos e conceituais relativos aos problemas da produtividade e crescimento econômico. Na seção seguinte, apresenta-se a interpretação kaldoriana para o crescimento da produtividade e formula-se a especificação da lei KV. Na seção três, descreve-se o procedimento operacional e na quarta, os resultados e análise. Finalmente, na parte cinco, apresentam-se as considerações finais. 


\section{Modelo teórico e analítico}

As idéias inerentes à Lei Verdoorn sugerem a existência de uma associação estável e constante entre taxa de crescimento da produtividade da mão-de-obra e taxa de crescimento da produção em cada setor da economia. Verdoorn (1980), ao analisar a relação entre a produtividade do fator trabalho e o nível da produção, usou dados em painel de diferentes países. Os pressupostos básicos do modelo de Verdoorn partem do princípio de que o setor industrial emprega toda a sua força de trabalho disponível a dada taxa de salários, conforme os estereótipos clássicos de mercado de trabalho, aduzindo que a oferta de trabalho é determinada pela taxa de salários existentes em cada setor e que esta guarda relação de proporcionalidade com a produtividade. Com base nessas proposições, o aumento na produtividade causaria aumento nos salários, o que determinaria o fluxo da mão-de-obra de outros setores no sentido dos de maior taxa de salários, principalmente a migração do setor agropecuário para o industrial. Desse modo, explica-se a tendência de aumento na taxa de ocupação no setor industrial em relação aos outros setores da economia.

O fundamento matemático e teórico de Verdoorn pode ser descrito com base em Rowthorn (1979). Parte de uma função de produção,

$Y=f(E)$,

em que $\mathbf{Y}$ é quantidade de produto e $\mathbf{E}$, nível de emprego. A partir daí, tem-se a expressão (2) da produtividade média, cujos rearranjos estabelecem a relação entre a produtividade média e a função de produção original em (3).

$$
\frac{Y}{E}=\frac{f(E)}{E},
$$




$$
\frac{Y}{E} E=f(E) .
$$

Tomando os logaritmos de (2) e diferenciado o resultado pode ser escrito pela expressão 5, em que $\mathbf{p}$ é taxa de crescimento da produtividade e e, taxa de crescimento de emprego.

$$
\begin{aligned}
& \operatorname{Ln}\left(\frac{Y}{E}\right)+\operatorname{Ln}(E)=\operatorname{Ln} Y . \\
& \mathbf{p}+\mathbf{e}=\mathbf{y} .
\end{aligned}
$$

Logo, o crescimento do produto $\mathbf{y}$ é dado pela parcela de crescimento da mão-de-obra (e) e pela parcela de crescimento da produtividade (p). Com base na relação emprego-produtividade, que é a própria curva de oferta de trabalho na equação (6), e substituindo-a na identidade $\mathbf{y}=\mathbf{p}+$ e, obtém-se (7),

$$
\begin{aligned}
& e=-\frac{\alpha}{\beta}+\frac{1}{\beta} p, \\
& y=\left[-\frac{\alpha}{\beta}+\frac{1}{\beta} p\right]+p,
\end{aligned}
$$

cujos rearranjos com $\beta y=-\alpha+p+\beta p$ e $\beta y=-\alpha+(1+\beta) p$ resultam na expressão (8),

$$
p=\frac{\alpha}{1+\beta}+\frac{\beta}{1+\beta} y,
$$

que é a relação entre o crescimento da produtividade e o crescimento da produção setorial. Implícita à Lei Verdoorn e conforme Routhorn (1979), a relação linear entre produtividade e produção é determinada pelas 
condições de oferta de trabalho, independentemente da tecnologia. Para completar o modelo, resta especificar a tecnologia de produção e uma função de investimento a ela associada. Admitindo a tecnologia do tipo Cobb-Douglas, tem-se (9):

$Y=E^{\alpha} K^{\beta}$

Se $\left[\frac{K}{E}\right]=k$ for o nível de capital por trabalhador usado em determinado setor, obter-se-á a expressão (10), abaixo,

$$
Y=E^{\alpha} E^{\beta} k^{\beta},
$$

que, em termos de variação, resulta em (11) ou (12).

$$
\begin{aligned}
& \frac{\Delta Y}{Y}=(\alpha+\beta) e+\beta k, \\
& \stackrel{*}{Y}=(\alpha+\beta) \stackrel{*}{e}+\beta \stackrel{*}{k} .
\end{aligned}
$$

A equação (12) é dada pelas taxas de crescimento da renda, emprego e capital. Substituindo (12) em (5), após sucessivos arranjos, tem-se a equação (13).

$$
p=\left[\frac{(\alpha+\beta-1)}{(\alpha+\beta)} y+\frac{\beta}{(\alpha+\beta)} k\right]
$$

que expressa a relação entre crescimento da produtividade, produção e capital. Conforme Rowthorn (1979), a expressão (13) não estabeleceu relação direta entre produtividade e renda, uma vez que o estoque de capital $\mathbf{k}$ não havia sido definido. O problema reside na falta de 
especificação de uma função investimento. Nesse caso, se o estoque de capital setorial crescer à taxa $\boldsymbol{\theta}$, o nível de capital por trabalhador crescerá à taxa $\mathbf{k}=\boldsymbol{\theta}-\mathbf{e}$, que, substituído em (13) e sabendo que o crescimento de emprego resulta da diferença entre crescimento da produção e crescimento da produtividade, $\mathbf{e}=\mathbf{y}-\mathbf{p}$, tem-se:

$$
p=\left[\frac{(\alpha+\beta-1)}{(\alpha+\beta)} y+\frac{\beta}{(\alpha+\beta)} k(\theta-p-y)\right] .
$$

O desenvolvimento da expressão (14) e suas modificações resultam em(15).

$$
p=\frac{\beta}{\alpha} \theta+\frac{(\alpha-1)}{\alpha} y .
$$

A relação entre $\mathbf{p}$ e $\mathbf{y}$ é chamada de elasticidade da produtividade em relação à renda, aqui na ótica do PIB. Ela é interpretada como a proporção de aumento na produção, que se deve ao aumento percentual na produtividade de trabalho. $\mathrm{O}$ resultado dessa relação evidencia a tecnologia de produção e a existência de retornos crescentes à escala. Essas derivações analíticas ilustram o suporte empírico para a demonstração da Lei Verdoorn, como apresentada na fórmula (16).

$\mathbf{p}=\mathbf{a}+\mathbf{b y} ; \mathbf{b}>\mathbf{0}$,

ou, ainda, conforme Kaldor (1975) e Leon-Ladesma (1998), tem-se a expressão abaixo, em que $\boldsymbol{\alpha}, \boldsymbol{\beta}, \boldsymbol{\theta}$ e $\boldsymbol{\gamma}$ são parâmetros a serem estimados: $\mathbf{e}=\boldsymbol{\theta}+\boldsymbol{\gamma} \mathbf{y} ; \mathbf{0}<\boldsymbol{\gamma}<\mathbf{1}$. Como, por definição, $\mathbf{p}=\mathbf{q}-\mathbf{e}$, deve haver boa correlação entre p e q. Isso porque, na perspectiva kaldoriana, a principal restrição ao crescimento recai sobre a demanda das exportações e não sobre a oferta de emprego, de forma a dar consistência ao tratamento de emprego como variável exógena em relação à produção e produtividade. Neste sentido, Kaldor (1975) obteve resultados não- significativos para a equação de Verdoorn, nos setores agrícola e comercial. No caso do setor industrial, a elasticidade da produtividade em relação ao PIB, o 
coeficiente de $\mathbf{p}$ em relação à $\mathbf{q}$ e de $\mathbf{e}$ em relação à $\mathbf{q}$, foi estimada em 0,50 , o que sugere relação positiva e significativa entre $\mathbf{p}$ e e e comprova ${ }^{5}$ a verificação da lei Verdoorn.

\subsection{Especificação do modelo}

O que se pretende observar é a existência de relação de longo prazo entre produtividade de trabalho e produto setorial, já que, no curto prazo, se sabe também da relação conhecida como a lei de Okun, que é dada pelos hiatos de produto e desemprego. Partindo da identidade $\mathbf{y}=\mathbf{p}+\mathbf{e}$, dada pela variação ao longo do tempo, deduz-se que $\mathbf{p}=\mathbf{y}-\mathbf{e}$. A especificação kaldoriana, a ser estimada pelo Método dos Mínimos Quadrados Ordinários, é dada em (17) ou (18), com u, o termo aleatório e suas propriedades características:

$$
\begin{aligned}
& \mathbf{p}=\mathbf{b}_{0}+\mathbf{b}_{1} \mathbf{y}+\mathbf{u}, \\
& \mathbf{e}=-\mathbf{a}_{0}+\mathbf{a}_{1} \mathbf{y}+\mathbf{u},
\end{aligned}
$$

admitindo que $\boldsymbol{\beta}_{1}>0 ; 0<\alpha_{1}>1$ e que $\boldsymbol{\beta}_{0}=-\mathbf{a}_{0}$ e $\boldsymbol{\beta}_{1}=\left(1-\mathbf{a}_{1}\right)$, por definição, o crescimento da produtividade é dado pela diferença entre o crescimento do produto e o de emprego. Os interceptos $\boldsymbol{\beta}_{\mathbf{0}}$ e $\boldsymbol{\alpha}_{\mathbf{0}}$ refletem o progresso tecnológico autônomo e, portanto, exógeno ao setor e admite-se que seu sinal seja negativo, para mostrar que, ao nível da produção zero, o crescimento de emprego no setor deve cair por causa dos avanços técnicos gerados nos outros setores da economia. A elasticidade-emprego, em relação ao produto setorial $\alpha_{1}$, e a elasticidade-produtividade, em relação ao produto $\boldsymbol{\beta}_{1}$, definem o tamanho e a natureza de retornos. Pieper (2001) preferiu a especificação (19), em vez da (18), pois, como argumentou, a correlação entre $\mathbf{p}$ e $\mathbf{y}$ seria espúria, uma vez que, com $\mathbf{p}=\mathbf{q}-\mathbf{e}$, se $\mathbf{e}=\mathbf{0}$ ou constante,

Considerando a produção como uma variável exógena e determinada pela demanda, qualquer erro estaria associado ao termo emprego. 
deveria haver correlação perfeita entre p e y. A expressão (19) representa a forma simples da Lei Verdoorn ${ }^{6}$, para averiguar a existência de retornos à escala, em nível setorial.

Testam-se as hipóteses de que $\boldsymbol{\beta}_{1}$ seja positivo e que $\mathbf{0}<\boldsymbol{\alpha}_{1}<\mathbf{1}$. Esta última condição é interpretada como evidência da existência de retornos crescentes. Por outro lado, se $\boldsymbol{\alpha}_{\mathbf{1}}=\mathbf{1}$, há evidência de retornos constantes à escala. Como propôs Bianchi (2001), usa-se o modelo de ajustamento parcial na forma $g_{n}=-\eta+\rho g_{y}+\sigma g_{n(t-1)}$, em que $\rho$ é interpretado como a resposta de emprego à produção no curto prazo, enquanto $\rho /(1-\sigma)$, como elasticidade de longo prazo.

\subsection{Fonte e tratamento de dados}

A base de dados usada consistiu das séries do Instituto de Pesquisas Econômicas Aplicadas e do Instituto Brasileiro de Geografia e Estatística sobre estatísticas brasileiras sobre emprego e PIB agregados para agropecuária, indústria e serviços. A interpretação dos resultados baseouse na teoria da elasticidade, pela qual se determina a elasticidade de emprego em relação à taxa de crescimento da renda setorial. Para levar em conta o componente de tendência para flutuação cíclica do desemprego e produção, os dados anuais foram tratados na base de médias móveis em três períodos, para eliminar possíveis efeitos sazonais. Todos os dados foram tratados pelos respectivos valores logarimizados.

Conforme Bianchi (2001), inicialmente, os trabalhos que visavam testar a lei Verdoorn eram baseados em dados de seção-cruzada entre diferentes países e regiões. Entretanto, este procedimento foi progressivamente abandonado em favor de análises em seção-cruzada, em nível regional ou em série temporal, por causa de problemas de correlação espúria relativos a regiões com diferentes características tecnológicas. No caso de séries em seção-cruzada regional, mesmo quando existiam fortes

6 Outras especificações da Lei Verdoorrn podem ser observadas por $\mathrm{y}=\mathrm{a}+\mathrm{be}$, em que o crescimento do produto depende do crescimento do nível de emprego. 
dualismos em termos de desenvolvimento, o uso da abordagem em série temporal era recomendado. A plotagem gráfica do PIB foi a forma preliminar usada para a análise da estacionariedade (Lamounier e Leite, 2004). Conforme Gujarati (1995), para verificar se as séries do PIB são não-estacionárias, ajusta-se o modelo $y_{t}=\rho y_{t-1}+v_{t}$; para testar a hipótese de $\mathrm{r}$ ser estatisticamente igual a 1 ou equivalente, estima-se $\Delta y_{t}=(\rho-1) y_{t-1}+v_{t}=\delta y_{t-1}+v_{t}$ para testar a nulidade de d. Em termos práticos, realizou-se o teste de Dickey-Fuller nas versões simplificadas tipo $\Delta y_{t}=\delta y_{t-1}+v_{t}, \quad \Delta y_{t}=\beta_{1}+\delta y_{t-1}+v_{t}$, $\Delta y_{t}=\beta_{1}+\beta_{2} t+\delta y_{t-1}+v_{t}$ (Gujarati, 1995). O teste Dickey-Fuller Aumentado visou eliminar o efeito do fator tendência e verificar o impacto líquido dos termos defasados. Finalmente, realiza-se o teste de causalidade de Granger, para confirmação da relação causal entre produtividade e renda, pois Rowthorn (1975) e Richard et al. (1998) questionaram a especificação apropriada para a Lei Verdoorn, se produção y ou se produtividade $\mathbf{p}$ em relação ao emprego e. Para descrever as alterações estruturais no emprego em cada setor, recorreu-se ao índice de mudança estrutural sugerido em Cavalheiro (2002) e definido por $I M E=\frac{\sum\left(e_{i, t}-e_{i, t-1}\right)}{2}$ em que $\mathrm{e}_{\mathrm{i}, \mathrm{t}} \mathrm{e}_{\mathrm{i}, \mathrm{t}-\mathrm{r}}$ representam a participação da população empregada em cada setor, em relação ao total da população ocupada, nos períodos t e t-1, respectivamente. O IME é uma extensão de que os setores estão crescendo e o valor 0 traduz mudança estrutural nula e valor 100, mudança estrutural máxima. 


\section{Resultados e discussões}

\subsection{Participação setorial do pessoal ocupado na economia}

A Figura 1 ilustra o comportamento setorial do pessoal ocupado na economia brasileira. Observa-se que, em média, registrou-se queda na participação do pessoal ocupado no setor agropecuário em detrimento da expansão da taxa de ocupação no setor de serviços, pois o setor industrial apresentou-se ligeiramente estável, oscilando entre 19\%, em 2003, e 23\%, em 1990. No entanto, a expansão do nível de ocupação no setor de serviços, embora historicamente superior no horizonte 19902003 , mostrou-se crescente, visto que passou de $51 \%$ a $62 \%$ no período, o que demonstrou ser a base da absorção de grande parte do trabalho ativo na economia. Do início do Plano Real até finais de 2003, a taxa de participação aumentou, em média, $8 \%$ ao ano, ou seja, houve absorção média de $0,1 \%$ ao ano. De 1990 a 2003, a taxa de crescimento da população ocupada, em nível nacional, foi de $1,02 \%$, enquanto na agropecuária a população ocupada decresceu 1,82\% em média, por ano. O setor de serviços, comprovada a sua força de absorção, registrou expansão de mão-de-obra em $2,53 \%$, o que compensou o fraco desempenho da agropecuária e da indústria (-0,036\%). Já no setor da agropecuária observou-se cenário oposto, visto que a participação do fator trabalho no nível de ocupação total caiu lentamente ao longo do tempo e, em média, de $0,06 \%$ ao ano. Entretanto, a Figura 2 mostra que o número total de pessoas ocupadas aumentou em, aproximadamente, $0,92 \%$ durante o período, enquanto na agropecuária o comportamento foi inverso, de queda anual de $0,88 \%$. No setor industrial, observou-se queda incipiente, de comportamento negativo, de $0,04 \%$, portanto, houve evolução, de grande resistência, na absorção de mão-de-obra neste setor. 
Henrique Tomé da Costa Mata, Niraldo José Ponciano \& Paulo Marcelo de Souza

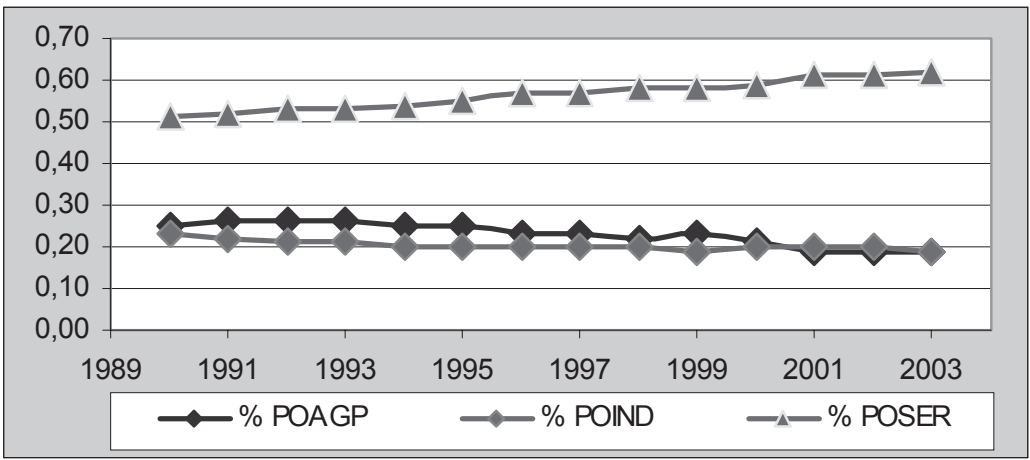

Figura 1 - Comportamento setorial do pessoal ocupado na economia.

O nível de ocupação no setor de serviços aumentou, em média, 1\%, o que compensou também a queda relativa na agropecuária. Esses resultados demonstram que a agropecuária foi pouco dinâmica na geração de emprego, apesar dos efeitos multiplicadores setoriais de impactos freqüentemente revelados neste setor.

\subsection{Comportamento do PIB, valor adicionado e produtividade setorial}

O PIB real cresceu à taxa média anual de 2,56\%, de 1990 a 2003 , enquanto a produtividade média da mão-de-obra aumentou apenas $1,54 \%$. Com base na Figura 2, pode-se inferir que a velocidade de absorção de mão-de-obra na economia foi maior que a própria evolução do PIB, de maneira que o comportamento da produtividade que decorreu deles foi mais lento na sua tendência, em termos gerais. Na Figura 3, verifica-se que a evolução setorial do valor adicionado foi bastante semelhante, embora o valor adicionado da agropecuária se mantivesse dentro dos limites de 200 bilhões de reais. 


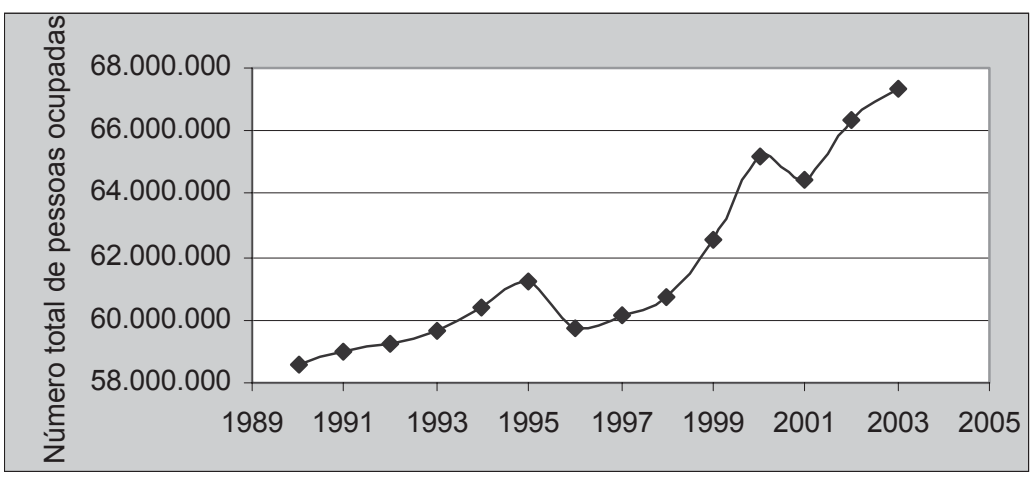

Figura 2 - Evolução do número total de pessoas ocupadas na economia brasileira.

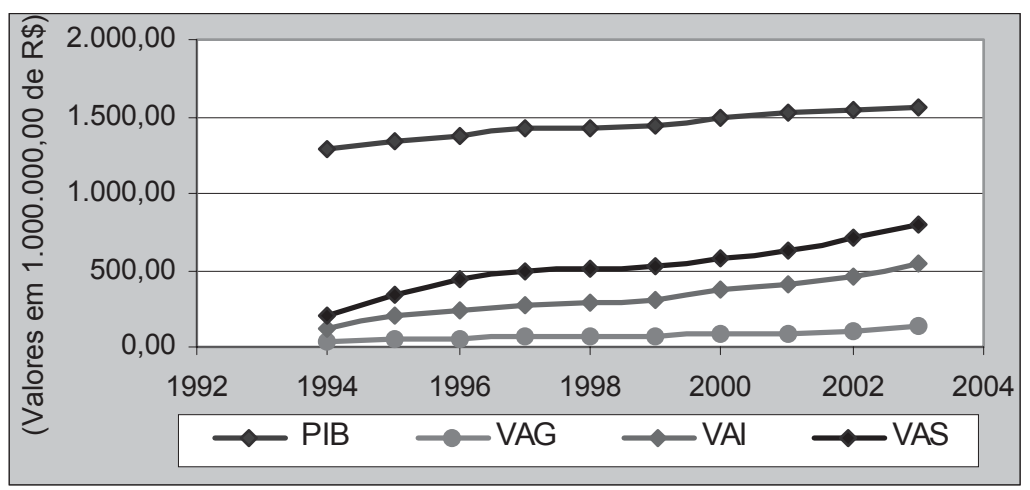

Figura 3 - Comportamento setorial do PIB e dos valores adicionados na economia brasileira.

Quando se consideram os dados a partir do Plano Real, que justificam a mudança estrutural que ocorreu na economia nos períodos pré e pós Plano, constata-se que a população ocupada cresceu 1,33\% em nível nacional, $0,83 \%$ no setor industrial e $2,82 \%$ no setor de serviços. Porém, na agropecuária houve queda de $2,23 \%$ na taxa de ocupação. Paralelamente a este comportamento setorial da população ocupada, em 
termos da produtividade, os dados apontaram ganhos de $0,70,15,28,12,67$ e 9,22\%, respectivamente, ao nível nacional, na agropecuária, indústria e serviços. Portanto, parte do ganho da produtividade que se registrou no setor agropecuário se deve aos choques tecnológicos intensivos em diferentes formas de uso de capital, visto que a expansão da produção esteve associada à redução na intensidade de uso da mão-de-obra.

\subsection{Estimativa do modelo de Kaldor-Verdoorn}

Com base na análise do correlograma e no teste da raiz unitária de DickeyFuller Aumentado, as séries do PIB e a produtividade média de emprego caracterizaram-se pela não-estacionariedade. Porém, as séries de produtividade média setorial para a agropecuária, indústria e serviços foram todas estacionárias, apresentando, respectivamente, valores de ADF de 9,13; 29,87 e -34,98, comparados aos valores críticos de MacKinnon, de 5,47. Como o ajustamento de duas séries nãoestacionárias, uma em relação à outra, torna o valor dos parâmetros enviesados, no que se considera de regressão espúria, estimaram-se os parâmetros associados à primeira diferença do PIB e da produtividade, obtendo-se valores críticos de MacKinnon elevados para o teste do ADF. 
Tabela 1 - Resultados setoriais estimados para crescimento da produtividade e valor adicionado e população ocupada na economia brasileira, 1994-2003

\begin{tabular}{llll}
\hline \multicolumn{1}{c}{ Setores } & \multicolumn{1}{c}{$\begin{array}{c}\text { Produtividade } \\
(\%)\end{array}$} & $\begin{array}{c}\text { PIB e Valor } \\
\text { Adicionado (\%) }\end{array}$ & $\begin{array}{c}\text { População } \\
\text { Ocupada (\%) }\end{array}$ \\
\hline Nacional & 0,70 & 2,03 & 1,33 \\
Agropecuária & 15,28 & 13,04 & $-2,23$ \\
Indústria & 12,67 & 13,50 & 0,83 \\
Serviços & 9,22 & 12,04 & 2,82 \\
\hline
\end{tabular}

Fonte: Série de PIB a Preços de 2003 (Instituto de Pesquisas Econômicas Aplicadas). Séries de Valor Adicionado IBGE/SCN/(ANUAL).

O teste de causalidade de Granger, a $10 \%$ de probabilidade, resultou no valor $\mathrm{F}$ de 5,76 , o que indica que o crescimento do PIB não foi o fator determinante do crescimento da produtividade de trabalho e, contrariamente, os dados estimados apontaram rejeição da hipótese nula, para a estatística $\mathrm{F}$ de 0,53 , de que o crescimento da produtividade não é a força causadora do crescimento do PIB. Desse modo, admitiu-se a relação unidirecional entre produtividade e PIB, na ótica da renda. A variável emprego mostrou-se independente tanto em relação à produtividade quanto em relação ao PIB, o que apóia a hipótese kaldoriana de que é o comportamento do PIB que determina o comportamento da produtividade do trabalho, e não o contrário.

Os resultados obtidos do ajuste das diferenças logarítmicas anuais da produtividade média de trabalho, Produto Interno Bruto e Valor Adicionado são apresentados na Tabela 2, considerando agregados nacionais da produção, emprego e agregados setoriais na agropecuária, indústria e serviços. Ao usar o procedimento econométrico dos Mínimos Quadrados Ordinários para estimação dos parâmetros da relação entre o crescimento 
da produtividade e crescimento do produto, observou-se que todos os coeficientes $\boldsymbol{\beta}$ 's estimados foram positivos, pelo teste de restrição linear de Wald, e estatisticamente significativos, pelo teste t, o que confirma a interdependência da produção e produtividade.

Em nível agregado nacional, a elasticidade de curto prazo da produtividade em relação à produção foi estimada em, aproximadamente, 0,82 , o que indica que o impacto do crescimento de um ponto percentual na renda, no curto prazo, implica a elevação em cerca de $0,8 \%$ no nível da produtividade nacional. No longo prazo, foi obtida a elasticidade de valor igual a 1,11, que mostra a evidência de que a produtividade aumenta em escala mais que proporcional ao aumento da produção. Naturalmente, uma das limitações do modelo pode ser compreendida das proposições teórico-matemáticas de Rowthorn (1979), sobre a definição e sobre os efeitos de capital e da natureza da função de produção agregada e tipo de retorno à escala. Desse modo, os valores da elasticidade estimados não são evidências efetivas do tipo de retorno à escala da economia, uma vez que as elasticidades não representam a resposta total da produção à intensidade de uso da mão-de-obra ocupada na economia. No nível agregado nacional, o coeficiente $\alpha$ teve valor negativo consistente com a teoria, pois, embora $\alpha$ represente o padrão tecnológico exógeno ao sistema, a hipótese é de que, ao nível de produção zero, espera-se que o crescimento do pessoal ocupado deva cair por razões da mobilidade e migração intersetorial na economia.

Em se tratando individualmente de cada setor, foi estimado um valor quase unitário de 0,99 para a elasticidade de curto e longo prazo e para a produtividade em relação ao valor adicionado no setor agropecuário, o que demonstra que a resposta da produtividade à produção no setor agropecuária foi relativamente maior em relação ao mesmo estímulo, em nível nacional. Vários estudos têm caracterizado o avanço tecnológico e a competitividade na agropecuária brasileira, na última década, com base na intensidade de uso de bens de capitais específicos, o que conferiu tecnicamente uma substituição de recursos humanos pelo fator capital. Essa dinâmica produtiva na agropecuária pode explicar, 
fundamentalmente, esse impacto da produção na elevação da produtividade de trabalho em relação aos outros setores, como se depreende da Tabela 2.

Quanto ao setor industrial, a elasticidade de curto prazo para a produtividade de trabalho, em relação à produção, apresentou valor estatisticamente não-significativo, o que mostra que a variação percentual da produtividade foi nula para choques de expansão na produção. $\mathrm{O}$ mesmo efeito se verificou para os resultados de longo prazo. Preliminarmente, a indicação é de que, dentro de certos limites, como o nível de agregação não permite captar os efeitos da intensidade de uso de capital, o impacto da produção sobre a produtividade de trabalho pode revelar-se nulo, em decorrência dos valores elevados da elasticidade de produção em relação ao fator capital.

Já o setor de serviços apresentou elasticidade unitária tanto no curto quanto no longo prazo. Comparativamente ao setor industrial, o setor de serviços mostrou-se absolutamente mais dinâmico no impacto do incremento da produção sobre a produtividade de emprego, podendo-se afirmar o mesmo para o setor da agropecuária. 
Tabela 2 - Resultados do ajuste de um modelo, baseado na Lei KV, à economia brasileira

\begin{tabular}{lllll}
\hline Setores & Intercepto & Elasticidade & $\mathbf{R}^{2}$ & $\mathbf{F}$ e DW \\
\hline & $\alpha=-0,0102$ & $\beta=0,8158$ & & \\
NACIONAL & $\mathbf{t}=-1,2100$ & $\mathbf{t}=2,9114$ & 0,58 & $\mathbf{F}=6,27$ \\
& Erro $(\mathbf{E})=0,008$ & $\mathbf{E}=0,2799$ & & $\mathbf{D W}=2,04$ \\
\hline AGROPECUÁRIA & $\mathbf{t}=0,9945$ & $\mathbf{t}=52,93$ & 0,99 & $\mathbf{F}=2983$ \\
& $\mathbf{E}=0,0206$ & $\mathbf{E}=0,01872$ & & $\mathbf{D W}=2,29$ \\
\hline INDÚSTRIA & $\alpha=1,4688$ & $\boldsymbol{\beta}=3,69 \mathrm{E}-09^{\mathbf{n s}}$ & & \\
& $\mathbf{t}=1,6455$ & $\mathbf{t}=1,6480$ & 0,62 & $\mathbf{F}=9,09$ \\
& $\mathbf{E}=0,8926$ & $\mathbf{E}=2,24 \mathrm{E}-09$ & & $\mathbf{D W}=1,54$ \\
\hline SERVIÇOS & $\alpha=-0,0268$ & $\boldsymbol{\beta}=1,0055$ & & \\
& $\mathbf{t}=-3,9588$ & $\mathbf{t}=137,43$ & 0,99 & $\mathbf{F}=25836$ \\
& $\mathbf{E}=0,0067$ & $\mathbf{E}=0,0073$ & & $\mathbf{D W}=2,32$ \\
\hline
\end{tabular}

Fonte: Resultados da pesquisa.

No período de 1994 e 2003, o crescimento do Valor Adicionado, nos três setores, apresentou poucas diferenças, enquanto a diferença no crescimento médio da população ocupada foi acentuada, com queda de $2,23 \%$ na agropecuária e aumento de $2,82 \%$ no setor de serviços, tendo o setor industrial absorvido crescimento de $0,83 \%$. Os dados alimentam a lógica de um aumento maior na produtividade de trabalho na agropecuária e na indústria, que teve menor absorção de emprego em relação ao de serviços. Entretanto, o setor de serviços apresentou menor desempenho em produtividade, o que pode ser explicado tanto pelo volume absoluto de seu valor adicionado quanto pelo fato de reter, também, grande parcela da força ativa empregada. 
A interpretação que Kaldor faz da Lei Verdoorn está baseada na estrutura teórica de "learning-by-doing", na linha de Arrow (1962), que estabelece a relação entre produtividade e choques cumulativos nos investimentos e na produção. Segundo ele, a experiência do processo de expansão da produção determina o desempenho da produtividade, pois esta tende a crescer quanto maior a produção. Embora se admita que a relação de causalidade da lei KV seja originalmente conhecida na direção da produção para a produtividade, concebe-se também que, em determinado momento do processo produtivo, os ganhos da produtividade podem sustentar aumentos na produção. A outra lógica inerente à interpretação kaldoriana consiste do efeito demanda, na perspectiva keynesiana. Choques de demanda podem determinar o nível da produtividade, evidência comprovada especialmente no crescimento das exportações agropecuárias em, aproximadamente, 13\%, de 1994 a 2003, em detrimento de queda de $7,51 \%$, ao ano, nas importações no período. Paralelamente, as exportações do setor industrial aumentaram somente 3,64\% no mesmo período, o que demonstra a dinâmica da agropecuária no crescimento econômico nacional.

A partir de 1994, com a recuperação da produção industrial após o plano de estabilização macroeconômica, não houve expansão efetiva de emprego na economia, devido às mudanças estruturais que ocorreram, principalmente, na fonte da estrutura organizacional, da produção e do mercado de trabalho. Em relação à análise das mudanças na estrutura de emprego, o setor da agropecuária apresentou alteração muito incipiente, com valor baixo de 9,1, para uma escala máxima de 100, no caso de uma mudança estrutural completa. No setor industrial, a estrutura e a participação do emprego mantiveram-se praticamente as mesmas, pois o valor calculado, em torno de 9,6, indicou mudança estrutural baixa também. Entretanto, foi no setor de serviços que se verificou mudança expressiva na estrutura de emprego. $\mathrm{O}$ valor calculado para o índice de mudança estrutural no setor de serviços, em torno de 31 , sinalizou alteração efetiva na estrutura de emprego neste setor. 


\section{Conclusões}

A Lei KV, ao associar o desenvolvimento da produtividade ao crescimento econômico, fundamenta-se na noção de causação circular cumulativa. Com bases neste pressuposto, setores mais pobres e mais ricos tenderiam a se distanciar cada vez mais do desenvolvimento, porque os setores mais atrativos receberiam maiores estímulos dos investimentos, gerando um processo de círculo vicioso que afastaria o sistema de suas condições de equilíbrio. Entretanto, não obstante as incursões teóricas intrínsecas à lei, ela permite observar o tipo de relação de causalidade existente entre produtividade do trabalho e níveis da produção. Os resultados obtidos da estimação dos parâmetros associados a tal relação comprovaram o teste da evidência da lei para a economia brasileira, à exceção do setor industrial, que apresentou as elasticidades marginais da produtividade de trabalho nulas, no curto e longo prazos. Devido à natureza agregada dos dados, esse comportamento da medida de impacto pode ser explicado pela estrutura especial da função da produção agregada no setor industrial e de suas elasticidades de produção para diferentes tipos de capital. Portanto, o crescimento da produtividade do trabalho não se deveu ao crescimento do produto no setor industrial. Com base na análise dos trabalhos nesta área específica e das referências existentes, novas pesquisas sobre a aplicação da lei KV devem ser realizadas, na expectativa de consolidar a sua verificação empírica na economia brasileira. 


\section{Referências}

ARROW, K. J. The economic implication of learning by doing. Review of Economics Studies, vol. 14, 1962, pp. 155-73.

BIANCHI, C. A rappraisal of Verdoor's Law for the Italian Economy: 1951-1997. Dipartimento di economia politica e metodi quantitative. Università degli studi di Pavia, Via San Felice, 5 I-27100 Pavia, Aprile, 2001.26p.

CAVALHEIRO, N. População ocupada e mobilidade: um enfoque setorial sobre o Brasil nos anos 90. Anais XIII Encontro da Associação Brasileira de Estudos Populacionais. Ouro Preto, Minas Gerais, 4-8 de outubro, 2002, 15p.

GONÇALVES, E.; CASTRO, C. M.; MEDEIROS, T. R. Diferenciais de produtividade do trabalho no Brasil e o processo de catching up. Revista de Economia Contemporânea, Rio de Janeiro, 7 (2): 195 212, jul/dez. 2003.

GUJARATI, D. N. Basic Econometrics. New York: Third Edition, Mc Graw-Hill, Inc. 1995, 838 p.

KALDOR, N. Economic growth and the Verdoorn Law - a comment on Mr Rowthorn's Article. The Economic Journal, 85 (dec, 1975), 891 $-896$.

KALDOR, N. Productivity and growth in manufacturing industry: a reply. Economica, vol. 35, (November), 1968, pp. 385-91.

LAMOUNIER, W. M.; LEITE, C. A. M. Análise Estrutural de Séries Temporais. In: Métodos Quantitativos em Economia, Cap 20. Editores: Maurinho Luiz de Santos e Wilson da Cruz Vieira. Ed. UFV, p. 63-653, 2004 
LEON-LEDESMA, M. A. Economic growth and Verdoorn's Law in the Spanish Regions, 1962-1991. Journal of Economic Literature, JEL, classification number: O40, O47, R11. February, 1998. 25p.

PIEPER, Ute, Sectoral regularities of produtivities growth in developing countries - A kaldorian Interpretation. MERIT, Maastricht Research Institute on Innovation and Technology. The Netherlands, august, 2001,29p.

RICHARD I.D. H.; EUNICE L. Verdoorn's law and increasing returns to scale in the UK regions, 1968-91: some new estimates based on the cointegration approach. Oxford Economic Papers, v50, n2 (19), April 1998. p201.

ROWTHORN, R. E. A Note on Verdoorn's Law. The Economic Journal, 89 (March, 1979): 131- 133.

ROWTHORN, R. E. A reply to Lord Kaldor's Comment. The Economic Journal, 85 (December, 1975), 897 - 901.

ROWTHORN, R. E. What remains of Kaldor Laws? Economic Journal, 85, 10-19. 1975.

SALGADO, R. Produtivity Growth in Canadá and the United States. Finance \& Development (F\&D), Dec, p. 1 - 8., 2000.

VERDOORN, P. J. Verdoorn's law in retrospect: a comment. Economic Journal, vol, 90 (june), 1980, p. 382-5.

VIEIRA, E. T. A produtividade e emprego nos anos 90: o debate no Brasil. Dissertação de Mestrado, Universidade Mackenzie, Curso de pós-graduação em economia. São Paulo, 1999. 


\begin{abstract}
An important indicator of the performance of any economy is the productivity and the connections between the labour market and the sectoral distribution of profit. Despite the restrictions inherent in the use of different concepts of productivity, Kaldor and Verdoorn have undertaken a serious verification of some relationship between production, employment and productivity, in an attempt to explain the dynamics of inter-regional and inter-sectoral economies, resulting in the designation of the so-called Kaldor-Verdoorn Law. The model for such a law asks questions about the relationship between the growth of labour productivity and product growth, throwing up scale indices of impact and return, in particular applicable to the industrial sector. The main theoretical bases of the model have been developed in this article, with empirical applications to the fields of GDP, employment and added value in the Brazilian economy, and the assertions maintained by Kaldor and Verdoorn in their estimates of the elasticities obtained have been proven. In general, an incipient alteration of the structure of sectoral employment was observed, except in the case of the service sector, which offered, on a scale of $1-100$, a change of 31 , and thus a labour market structure with a notable internal modification.
\end{abstract}

Keywords: GDP, productivity, Kaldor-Verdoorn Law. 